\title{
Capacity Analysis of Multicast Network in Spectrum Sharing Systems
}

\author{
Jianbo Ji*†, Wen Chen*\#, Haibin Wan*ł, and Yong Liu* \\ *Department of Electronic Engineering, Shanghai Jiaotong University, Shanghai, P.R, China 200240 \\ $\dagger$ Department of Electronic Engineering, Guilin College of Aerospace Technology, Guilin, P.R, China 541004 \\ $\ddagger$ School of Physical Science and Engineering Technology, Guangxi University, Nanning, P.R, China 530004 \\ \# SKL for Mobile Communications, Southeast University, China 211189 \\ Email: \{jijianbo; wenchen; dahai_good; yongliu1982\}@sjtu.edu.cn
}

\begin{abstract}
In this paper, we consider the capacity of a multicast network, where a single-antenna secondary base station (SBS) utilizes the spectrum licensed to primary users (PUs) to broadcast the same information to multiple secondary users (SUs) with a single-antenna simultaneously, when the interference received by PUs is less than a predefined threshold. Based on extreme value theory, we first derive the average capacity of the multicast/unicast schemes in the case that perfect channel station information from the SBS to the PUs (interference CSI) is known at the SBS. Due to limited cooperation between the SBS and the PUs, perfect interference CSI is not always available at the SBS. Thus, we also analyze the average capacity of the above-mentiontd schemes in the case of imperfect interference CSI at the SBS. The analytical and simulated results reveal that when interference CSI is perfectly available at the SBS, and the SBS transmit power $P$ is sufficiently larger than the interference temperature threshold $Q$, the average capacity of the multicast scheme scales as $\Theta(Q)$ similar to a previously known scaling law in the non-spectrum sharing multicast systems, while the average capacity of the unicast scheme scales as $\Theta(\log (1+P(\log N Q-\log \log N Q)))$ in the case of $Q \ll P \ll$ $N Q$ or $\Theta(\log (1+(N-1) Q))$ in the case of $P \gg N Q$, where $N$ denotes the number of SU. If perfect interference CSI is not available at the SBS, the average capacity of the multicast and unicast schemes will be respectively scaled by the factors $\frac{\lambda}{\lambda+1}$ and $\left|\frac{\lambda-1}{\lambda}\right|$, compared to that of perfect interference CSI available at the SBS, where $1 / \lambda$ denotes the mean of interference CSI estimation error.
\end{abstract}

\section{INTRODUCTION}

Cognitive radio (CR) technology has been proposed as promising solution to implement efficient reuse of the licensed spectrum by unlicensed devices [1], [2]. In an opportunistic spectrum access system, the secondary users (SUs) can only transmit in "white spaces", i.e., the frequency bands or time intervals where the primary users (PUs) are silent [1]; while in a spectrum sharing system, the SUs are allowed to transmit simultaneously with the active PUs, as long as the interference power from the SUs to the PUs is less than an acceptable threshold. Clearly the latter can achieve higher spectral efficiency at the expense of additional side-information at the SUs and increased signaling overhead. Recently there are many active studies on a spectrum sharing system recently [4], [5], [6]. The maximum allowable interference power at the PUs is called interference temperature [3], which guarantees the quality of service (QoS) of the PUs regardless of the SU's spectrum utilization.

On the other hand, there has been an increasing demand for the applications of sending a same information to multiple receivers. These applications often require highly reliable connections to all users, which are usually difficult to achieve in wireless networks. Multicasting, a special case of broadcasting, is considered to be an appropriate approach to solve this problem [7]. The Evolved Multimedia/Multicast service (E$\mathrm{MB} / \mathrm{MS}$ ) in the context of 3GPP/UMTS-LTE includes explicit provisions for point-to-multipoint physical layer multicasting [8]. Two conventional multicasting schemes have been extensively studied in the literatures [9], namely, the multicast scheme and unicast scheme. In the multicast scheme, the base station always transmits to all users at information decodable by the worst user, which throughput performance is limited by the worst channel user, and which capacity limits scales as $\Theta(1)$ [10]; while the unicast scheme exploits the multi-user diversity gain by broadcasting to the instantaneous best user only, which capacity scale as $\Theta(\log \log N)$ [10]. The multiple input and single output multicast asymptotical capacity limits has been investigated in [11], when the perfect CSI is available at the transmitter. It is shown that the adverse impact of the large number of users on the multicast capacity could be compensated by increasing the number of transmit antennas.

The capacity of multicast network in spectrum sharing systems may be different from that of non-spectrum sharing because interference regulation affects the transmit power of multicast base station. To our best knowledge, there has been no investigation on the capacity of multicast network in a spectrum sharing system. Based on this motivation, we investigate the effects on the capacity of multicast network in a spectrum sharing system where the secondary base station (SBS) restrictively utilize a licensed spectrum.

The rest of this paper is organized as follows. In Section II, we introduce the system model. In Section III, we derive the average capacity of the multicast scheme and the unicast scheme when perfect interference CSI is available at the SBS. In Section IV, the average capacity of the multicast scheme and the unicast scheme are derived when imperfect interference CSI is available at the SBS. In Section V, numerical simulation 


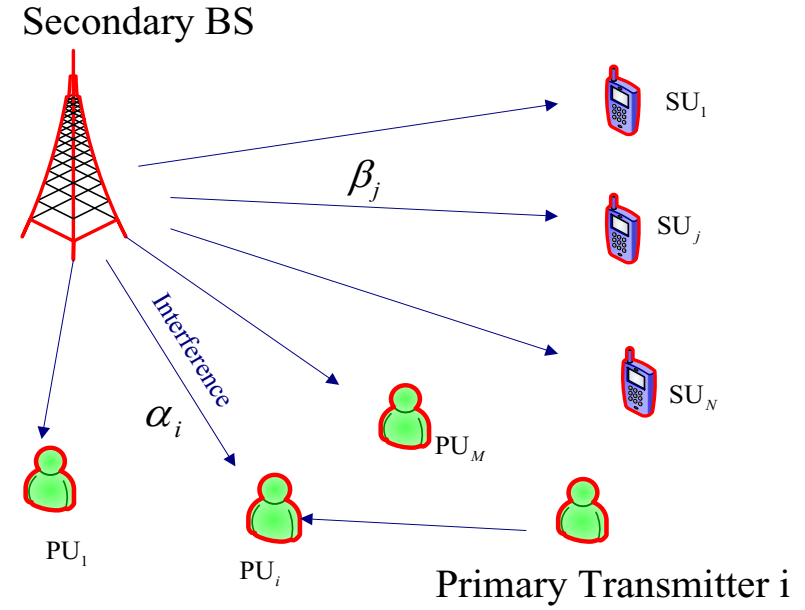

Fig. 1. System model

results are done to validate the theoretical results. Finally, conclusions are drawn in Section VI.

\section{SYSTEM MODEL}

As shown in Fig. 1, a spectrum sharing multicast network in a single-cell system is considered where a SBS utilizes the spectrum licensed to $M$ PUs to transmit the same information to $N$ SUs. All users are assumed to be equipped with a single antenna. In this system, it allows any information transmission from the SBS to SUs provided that the resulted interference power level at the PU is below some predefined threshold, which is known as the interference-temperature constraint $Q$ [2], [13], [18]. The interference-temperature represents the maximum allowable interference power level at the PU. The channel gains from the SBS to the PU $i$ and the SU $j$ are denoted by $\alpha_{i}$ and $\beta_{j}$, respectively, where $i \in\{1, \cdots, M\}$, $j \in\{1, \cdots, N\}$. The $\alpha_{i}$ and $\beta_{j}$ are assumed to be independent and identically distributed (i.i.d) exponential random fading channel. Assume that the SBS has the perfect information of SU's channel gains, $\beta_{j}$, all the time. Utilizing the feedback scheme, the SBS can obtain the interference CSI through periodic sensing of pilot signal from PUs by the hypothesis of channel reciprocity [12]. Then the SBS compute the maximum allowable transmit power $P_{t}$ according to $\alpha_{i}$ so as to satisfy the interference temperature constraint at the PU $i$. As in [4], we do not take into account primary transmitters here because the interference from primary transmitters can be translated into the noise term under an assumption that interference from the primary transmitter follows a white Gaussian distribution.

\section{Capacity of Multicast Network in Spectrum SHARING WITH PERFECT INTERFERENCE CSI}

Assume that the SBS is able to obtain the perfect interference channel gains $\alpha_{i}$ by feedback from the PUs [14], [15]. For simplifying mathematical analysis, $\alpha_{i}$ and $\beta_{j}$ are both assumed to be i.i.d exponential random variables with unit mean. The SBS allocates its peak power for transmission provided that the peak power is below the interference temperature. Otherwise, it adaptively adjusts its transmit power to the allowable level so that the interference received at the PUs is maintained as a given interference temperature level $Q$. Correspondingly, the transmit power of the SBS is given by [4]

$$
P_{t}=\left\{\begin{array}{l}
P, \alpha \leq \frac{Q}{P}, \\
\frac{Q}{\alpha}, \alpha>\frac{Q}{P},
\end{array}\right.
$$

where $P$ represents the peak power of the SBS transmission and $\alpha$ is defined as $\alpha \triangleq \max _{1 \leq i \leq M}\left(\alpha_{i}\right)$. Then the cumulative distribution function (CDF) and probability density function (PDF) of $\alpha$ are respectively given as

$$
F_{\alpha}(x)=\prod_{j=1}^{M}\left(1-e^{-x}\right), \quad f_{\alpha}(x)=M e^{-x}\left(1-e^{-x}\right)^{M-1} .
$$

The adjusted power of the SBS is used for sending the same information to the SUs. Thus, the received SNR $\gamma_{j}$ at the $j$-th $\mathrm{SU}$ is given by

$$
\gamma_{j}=\frac{P_{t} \beta_{j}}{\sigma^{2}}=\left\{\begin{array}{l}
P \beta_{j}, \alpha \leq \frac{Q}{P}, \\
\frac{Q \beta_{j}}{\alpha}, \alpha>\frac{Q}{P},
\end{array}\right.
$$

where the variance of white Gaussian noise is set to be 1 for analysis simplicity. Thus the $P$ and $Q$ can also be considered as the transmit SNR and the interference temperature to noise ratio, respectively. Then, the CDF of the received SNR from the $j$-th $\mathrm{SU}$ is

$$
F_{\gamma_{j}}(\gamma)=\operatorname{Pr}\left[\alpha \leq \frac{Q}{P}\right]\left(1-e^{-\frac{\gamma}{P}}\right)+\operatorname{Pr}\left[\frac{Q \beta_{j}}{\alpha} \leq \gamma \mid \alpha>\frac{Q}{P}\right] .
$$

From [4], we have

$$
\begin{aligned}
F_{\gamma_{j}}(\gamma)=(1 & \left.-e^{-\frac{Q}{P}}\right)^{M}\left(1-e^{-\frac{\gamma}{P}}\right)+M \sum_{k=1}^{M}\left(\begin{array}{c}
M-1 \\
k-1
\end{array}\right) \\
& \times(-1)^{k-1} e^{-\frac{Q k}{P}}\left[\frac{1}{k}-\frac{Q}{Q k+\gamma} e^{-\frac{\gamma}{P}}\right] .
\end{aligned}
$$

Then, the PDF of $\gamma_{j}$ can be derived as

$$
\begin{array}{r}
f_{\gamma_{j}}(\gamma)=\left(1-e^{-\frac{Q}{P}}\right)^{M} e^{-\frac{\gamma}{P}}+\frac{Q M}{P} e^{-\frac{\gamma}{P}} \sum_{k=1}^{M}\left(\begin{array}{c}
M-1 \\
k-1
\end{array}\right) \\
\times(-1)^{k-1} e^{-\frac{Q k}{P}}\left[\frac{P+Q k+\gamma}{P(Q k+\gamma)^{2}}\right]
\end{array}
$$

Based on this derivation, we will analyze the average capacity of the multicast and unicast schemes.

\section{A. Multicast Scheme}

In the multicast transmission scheme, the SBS always transmits to all SUs at information rate decodable by the SU with the worst channel. This scheme enables all the SUs to successfully decode the transmission. Therefore, the average capacity of the multicast scheme is given by [10]

$$
\begin{aligned}
C_{M} & \triangleq N E\left[\log \left(1+\gamma_{\min }\right)\right] \\
& =N \int_{0}^{\infty} \log (1+\gamma) f_{\gamma_{\min }}(\gamma) d \gamma,
\end{aligned}
$$


where $\gamma_{\min } \triangleq \min _{1 \leq i \leq N} \gamma_{i}$, whose probability density function is given by

$$
f_{\gamma_{\min }}(\gamma)=N f_{\gamma_{i}}(\gamma)\left(1-F_{\gamma_{i}}(\gamma)\right)^{N-1}
$$

Unfortunately, a closed form of (7) is not available, and it is difficult to fully understand the effects of the major parameters, such as $M$ and $N$, on the capacity by a numerical evaluation. We will, therefore, take a different approach to understand the asymptotic behavior of (7) in the scenario of large $N$.

Theorem 1: When $M=1$ and $P \gg Q$, the average capacity of the multicast scheme scales as

$$
C_{M}=\Theta^{1}(Q),
$$

as the number $N$ of $\mathrm{SU}$ goes to infinity.

Proof: Omitted due to limited space.

Theorem 1 indicates the capacity saturation in spectrum sharing systems is the same as that in the non-spectrum sharing system presented in [10]. This is because that the capacity of the multicast scheme is restricted by the received SNR of the SU with the worst channel. It also indicates that the capacity grows as the interference temperature $Q$ grows, this is because that the transmit power at the SBS increases with $Q$ as shown in (1).

\section{B. Unicast Scheme}

Now we consider the unicast scheme, where the SBS chooses the SU that has maximal received SNR. The average capacity of the unicast scheme is given by

$$
C_{U} \triangleq E\left[\log \left(1+\gamma_{\max }\right)\right]=\int_{0}^{\infty} \log (1+\gamma) f_{\gamma_{\max }}(\gamma) d \gamma,
$$

where $\gamma_{\max } \triangleq \max _{1 \leq i \leq N} \gamma_{i}$, whose PDF is given by

$$
f_{\gamma_{\max }}(\gamma)=N f_{\gamma_{i}}(\gamma) F_{\gamma_{i}}(\gamma)^{N-1}
$$

Theorem 2: When $M=1$ and $Q \ll P \ll N Q$, the average capacity of the unicast scheme scales as

$$
C_{U}=\Theta(\log (1+P(\log N Q-\log \log N Q))) ;
$$

if $P \gg N Q$,

$$
C_{U}=\Theta(\log (1+(N-1) Q)),
$$

as the number $N$ of $\mathrm{SU}$ goes to infinity.

Proof: Omitted due to limited space.

From Theorem 2, one can conclude that the average capacity of unicast scheme in spectrum-sharing scenario depends on the SBS transmit power $P$. When $Q \ll P \ll N Q$, the capacity scales as $\Theta(\log (1+P(\log N Q-\log \log N Q)))$. On the other hand, the capacity scales as $\Theta(\log (1+(N-1) Q))$, when $P \gg N Q$.

${ }^{1} f(n)=O(g(n))$ if and only if there are constant $c$ and $n_{0}$ such that $f(n) \leq c g(n)$ for any $n>n_{0} . f(n)=\Omega(g(n))$ if and only if there are constant $c$ and $n_{0}$ such that $f(n) \geq c g(n)$ for any $n>n_{0} . f(n)=$ $\Theta(g(n))$ if and only if there are constant $c_{1}, c_{2}$ and $n_{0}$ such that $c_{1} g(n) \leq$ $f(n) \leq c_{2} g(n)$ for any $n>n_{0}$.

\section{Capacity of Multicast Network in Spectrum SHARING WITH IMPERFECT INTERFERENCE CSI}

Due to limited cooperation between the SBS and the PUs, the accurate $\alpha_{i}$ is usually hard to be obtained at the SBS. Suppose that the channel from the SBS to the $j$-th PU is denoted as $h_{j}=\hat{h}_{j}+\Delta h_{j}$, where variable $\hat{h}_{j}$ and $\Delta h_{j}$ denote the perfect interference channel and the channel estimation errors, respectively. They are rayleigh distributed and independent of each other. Therefore, we can get $\alpha_{h_{j}}=\alpha_{\hat{h}_{j}}+\Delta \alpha_{h_{j}}$, where $\alpha_{\hat{h}_{j}}$ and $\Delta \alpha_{h_{j}}$ denote the magnitude square of $\hat{h}_{j}$ and $\Delta h_{j}$ respectively, which both follow exponential distribution. Assume that $\alpha_{\hat{h}_{j}}$ is normalized to have unit mean, and $\Delta \alpha_{h_{j}}$ 's mean value is $1 / \lambda(\lambda \neq 1)$.

\section{A. Multicast Scheme}

In this subsection, we asymptotically analyze the capacity to understand the effects of channel estimation errors in spectrum sharing environments. We can get the average capacity of multicast scheme using extreme value theory.

Theorem 3: When $M=1, P \gg \max (Q, \lambda Q)$, the average capacity of the multicast scheme under imperfect interference CSI available at the SBS scales as

$$
C_{M}=\frac{\lambda}{1+\lambda} \Theta(Q)
$$

as the number $N$ of SU goes to infinity.

Proof: Omitted due to limited space.

From Theorem 3, one can see that the average capacity of the multicast scheme under imperfect interference CSI available at the SBS is scaled by the factor $\frac{\lambda}{\lambda+1}$ compared to that with perfect interference CSI at the SBS shown in Theorem 1. This in fact implies that the channel estimation error will result in the average capacity loss, because the channel estimation error will leak interference power levels at the PUs.

\section{B. Unicast Scheme}

We now asymptotically analyze the capacity of unicast transmission scheme to understand the effects of channel estimation error on the average capacity. Based on extreme value theory, we get the average capacity of unicast scheme.

Theorem 4: When $M=1, \max (Q, \lambda Q) \ll P \ll N Q$, the average capacity of unicast scheme under imperfect interference CSI available at the SBS scales as

$$
C_{U}=\left|\frac{\lambda-1}{\lambda}\right| \Theta(\log (1+P(\log N Q-\log \log N Q))) ;
$$

if $P \gg N Q$, the average capacity scales as

$$
C_{U}=\left|\frac{\lambda-1}{\lambda}\right| \Theta(\log (1+(N-1) Q)),
$$

as the number $N$ of $\mathrm{SU}$ goes to infinity.

Proof: Omitted due to limited space.

From Theorem 4, it shows that the average capacity of the unicast scheme is scaled by the factor $\left|\frac{\lambda-1}{\lambda}\right|$ compared to that with perfect interference CSI shown in Theorem 2. 


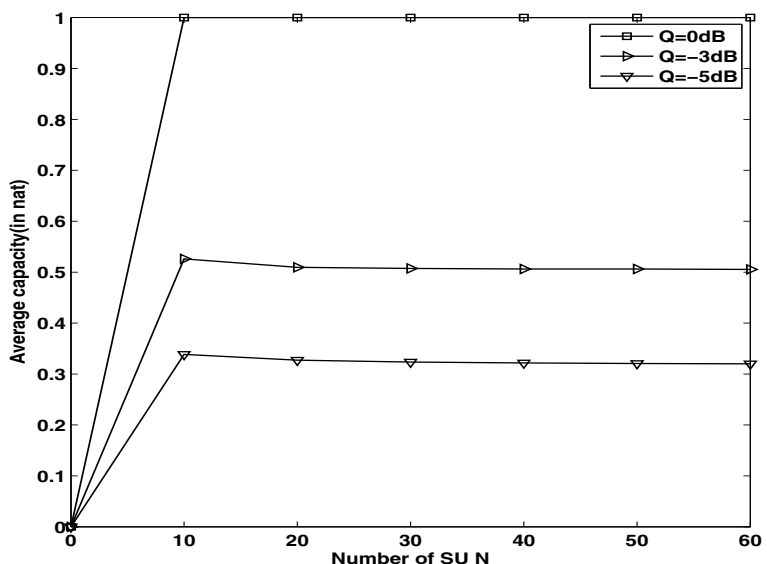

Fig. 2. Average capacity of multicast scheme versus the number of SU for different interference temperature $Q$

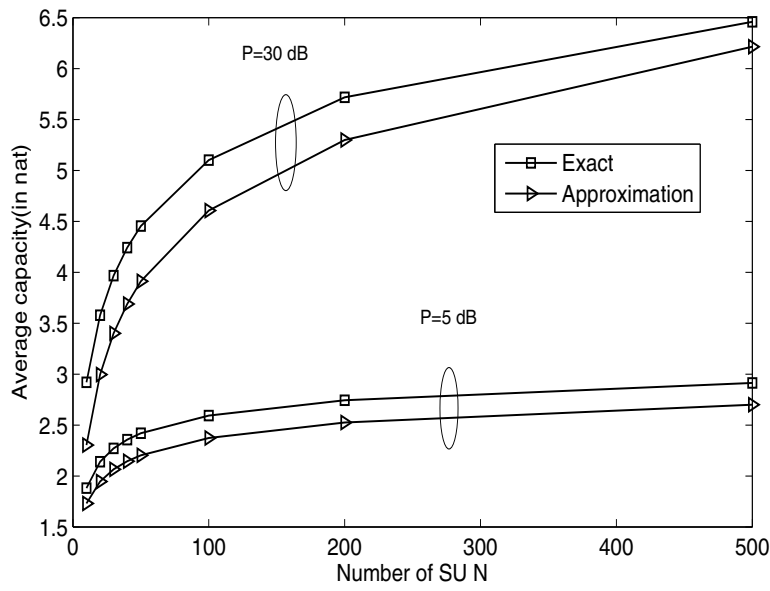

Fig. 3. Average capacity of unicast scheme versus the number of SU for two different transmit power $P$.

\section{NUMERICAL RESULTS}

Fig. 2 shows the average capacity of the multicast scheme versus the number $N$ of $\mathrm{SU}$ in spectrum sharing systems, when perfect interference CSI is available at the SBS. The SBS transmit power $P=20 \mathrm{~dB}$. In Fig. 2, there are three different interference temperature $Q$. When $Q=0 \mathrm{~dB}$, the capacity scales as $\Theta(1)$, which performance is similar to that in the non-spectrum sharing systems [10]. If $Q=-3 \mathrm{~dB}$, $5 \mathrm{~dB}$, the capacity scales as $\Theta(0.5), \Theta(0.3)$, respectively. The simulation result in Fig. 2 validates the asymptotic capacity presented in Theorem 1.

Fig. 3 shows the average capacity of the unicast scheme versus the number $N$ of $\mathrm{SU}$ for two different peak transmit power $P=5,30 \mathrm{~dB}$ for $Q=0 \mathrm{~dB}$. It is verified that the approximation results closely follow the exact simulations. It is also verified that a scaling law of capacity is $\log (1+(N-1) Q)$ when $P$ is sufficiently larger than $Q$. Fig. 3 also shows that the capacity grows very fast as $P$

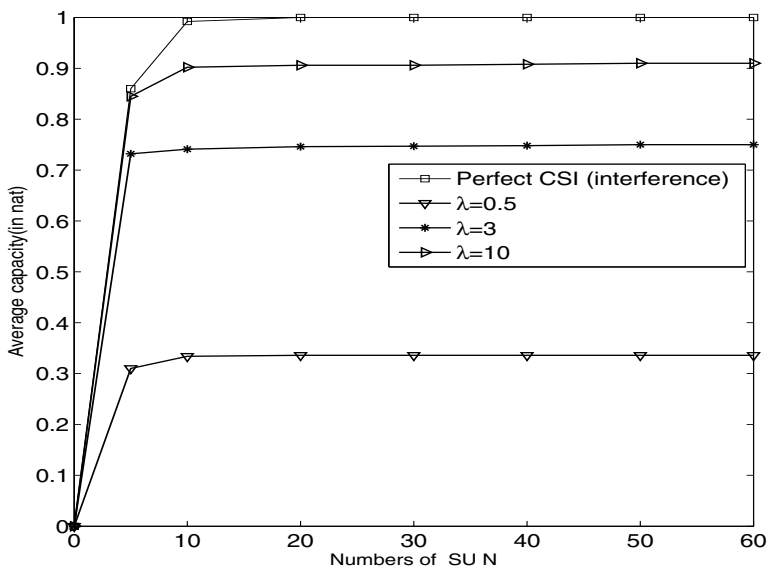

Fig. 4. Average capacity of multicast scheme versus the number of SU with imperfect interference CSI available at the SBS

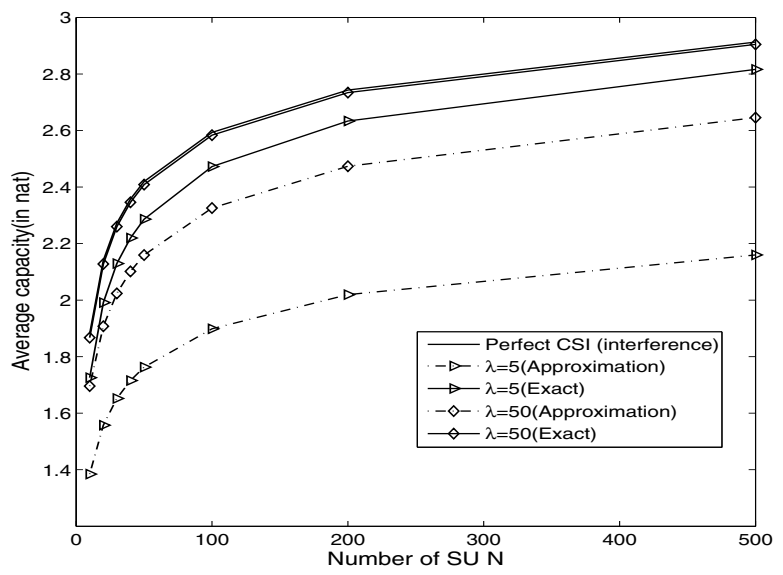

Fig. 5. Average capacity of unicast scheme versus the number of SU with imperfect interference CSI available at the SBS

becomes much larger such that $P \gg N Q$.

Fig. 4 shows the average capacity of the multicast scheme verus the number $N$ of SU for different $\lambda$ when the imperfect interference channel gain is available at the SBS. It shows that the channel estimation error will result in the average capacity loss. This is because that the channel estimation error will leak interference power levels at the PU. The average capacity loss will decrease as $\lambda$ increases since the large $\lambda$ means less channel estimation error. We further see that the average capacity loss approaches to zero when $\lambda$ increases.

Fig. 5 compares the average capacity of unicast scheme under the perfect and imperfect interference CSI available at the SBS. The SBS transmit power $P=5 \mathrm{~dB}, Q=0 \mathrm{~dB}$. It shows that the average capacity increases as $\lambda$ increases. this is because that the increasing of $\lambda$ results in the channel estimation error decreases. We further conclude that the average capacity loss approaches to zero when $\lambda$ goes to infinity. We also see that the asymptotic approximation exactly 


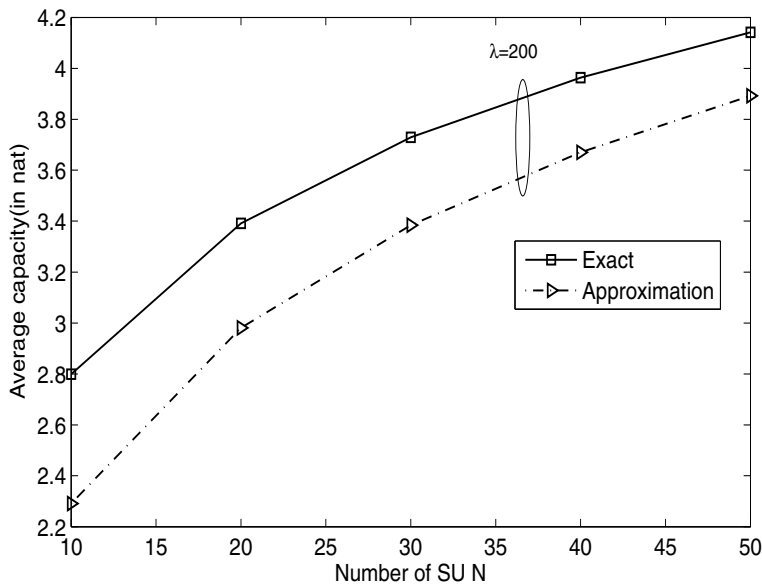

Fig. 6. Average capacity of unicast scheme versus the number of SU with high transmit power under imperfect interference CSI available at the SBS

characterizes the scaling law of the capacity, which scales as $\left|\frac{\lambda-1}{\lambda}\right| \Theta(\log (1+P(\log N Q-\log \log N Q)))$.

Fig. 6 shows the average capacity of the unicast scheme versus the number $N$ of $\mathrm{SU}$ when $P=30 \mathrm{~dB}, Q=0 \mathrm{~dB}$. It is verified that the asymptotic approximation exactly characterizes the scaling law of $\left|\frac{\lambda-1}{\lambda}\right| \Theta(\log (1+(N-1) Q))$, when $\lambda$ is sufficiently large.

\section{CONCLUSION}

In this paper, we analyze the average capacity of the cognitive multicast network where the SBS transmit power is regulated by a given interference temperature at the PUs. Based on extreme value theory, the asymptotic capacity of two multicast transmission schemes under the knowledge of the interference perfect CSI at the SBS is investigated. Due to limited cooperation between the SBS and the PUs, perfect interference CSI is hard to be obtained at the SBS. Therefore, we analyze the average capacity of the multicast and unicast schemes under the imperfect interference CSI available at the SBS, which are found to be respectively scaled by the factors $\frac{\lambda}{\lambda+1}$ and $\left|\frac{\lambda-1}{\lambda}\right|$, compared to those of perfect interference CSI available at the SBS.

\section{ACKNOWLEDGEMENT}

This work is supported by NSF China \#60972031, by SEU SKL project \#W200907, by Huawei Funding \#YJCB2009024WL and \#YJCB2008048WL, and by National 973 project \#2009CB824900.

\section{REFERENCES}

[1] J. Mitola, "Cognitive radio: An integrated agent architecture for software defined radio," Ph. D. dissertation, KTH, Stockholm, Sweden, December 2000.

[2] S. Haykin, "Cognitive radio:brain-empowered wireless communications," IEEE J. Select Areas Commun, vol. 23, no. 2,pp. 201-220, Feb. 2005.

[3] Federal Communications Commission, "Spectrum policy task force report," ET Docket No.02-135, Nov. 2002.
[4] T. W. Ban, W. Choi, B. C. Jung, and D. K. Sung, "Multi-User diversity in a spectrum sharing system," IEEE Trans. wireless commun., vol. 8, no. 1, pp. 102-106, Jan. 2009.

[5] R. Zhang, and Y. C. Liang, "Exploiting Multi-Antennas for Opportunistic Spectrum Sharing in Cognitive Radio Networks," IEEE J. Select Topics in Signal Processing, vol. 2, no. 1, pp. 88-102, Feb. 2008.

[6] L. Zhang, Y. C. Liang, and Y. Xin, "Joint Beamforming and Power Allocation for Multiple Access Channels in Cognitive Radio Networks," IEEE J. Select Areas Commun, vol. 26, no. 1, pp. 38-51, Feb. 2008.

[7] P. K. Gopala, and H. E. Gamal, "Opportunistic multicasting," in Proc. Asilomar Conference on Signals, Systems and Computers, November 2004.

[8] "Long term evolution(LTE): A technical overview," Motorla, Inc., Schaumburg, IL[online]. Available: http://business.motorola.com/ experiencelte/pdf/LTE\%20Technical\%200veriew.pdf

[9] M. Lopez, "Multiplexing,scheduling,and multicasting strategies for antenna arrays in wireless networks," Ph.D.dissertation, Massachusetts Institute of Technology, 2002.

[10] P. Kumar, and H. E. Gamal, "On the throughtput-delay tradeoff in cellular multicast," submitted to IEEE Trans.Inform.Theory.

[11] N. Jindaland, and Y. Q. Luo, "Capacity Limits of Multiple Antenna Multicast," Proc. IEEE Int. Symp. Information Theory (ISIT 06), Seatle, USA, July 9-14 2006.

[12] Q. Zhao, S. Geirhofer, L. Tong and B. M. Sadler, "Opportunistic spectrum access via periodic channel sensing," IEEE Trans.Signal Processing, vol. 56, no. 2, pp. 785-796, Feb. 2008.

[13] Website of FCC, http://www.fcc.gob/sptf/resports.html.

[14] A. Ghasemi and E. S. Soua, "Fundamental limits of spectrum-sharing in fading environments," IEEE Trans. Wireless. Commun, vol. 6, no. 2, pp. 649-658, Feb. 2007.

[15] J. M. Peha, "Approaches to spectrum sharing," IEEE Commun. Mag., vol. 43, no. 2, pp. 10-12, Feb. 2008

[16] B. C. Arnold, N. Balakrishnan, and H. N. Nagaraja A first course in order statistics., New York: John Wiley Sons, Inc., 1992.

[17] J. Pickands, "Moment convergence of sample extremes," Annals of Math.Statist., vol. 39, no. 3, pp. 881-889, 1968

[18] M. Gastpar, "On capacity under receive and spatial spectrum-sharing constraints," IEEE Trans. Inform. Theory., vol. 53, no. 2, pp. 471-487, Feb. 2007. 